\title{
Performance of the Upgraded Stacktail Momentum Cooling System in the Fermilab Antiproton Source
}

\author{
Ralph J. Pasquinelli, David McGinnis \\ Fermi National Accelerator Laboratory * \\ P.O. Box 500, Batavia, IL 60510
}

\begin{abstract}
Major changes in the Stacktail Momentum Stochastic Cooling system have resulted in an improved stacking rate as well as the capability to stack larger quantities of antiprotons. Both these effects result in higher initial and integrated luminosity for colliding beam physics. An over view of the changes and actual system performance will be presented.

\section{INTRODUCTION}

Since our initial report in 1987 [1], a number of changes have been made to this cooling system. It was discovered early in the history of the Antiproton source that excessive transverse core heating by the stacktail system was limiting the maximum core size. A subset of four of the twenty kickers was implemented as transverse kickers to minimize this effect. The original twenty kickers have been totally replaced with sixteen new planar loop design kickers.
\end{abstract}

Although the superconducting notch filters performed admirably through their life, there was the added expense of the liquid helium. These filters were replaced with Bulk Acoustic Wave (BAW) filters [2] that save approximately $\$ 200 \mathrm{~K}$ in annual operations costs. A system equalizer was also designed to maximize the system bandwidth. Finally, even with the new kicker design there is still core transverse heating. A special hybrid [3] has been used for the same subset of transverse kickers, but now these same kickers are both longitudinal and transverse.

\section{NEW PLANAR LOOP KICKERS}

The purpose of Stack Tail Momentum Kicker Upgrade was to minimize the betatron heating of the core due to the stack tail momentum stochastic cooling system. Previous to implementing the upgrade, the gain of the stack tail system had to be reduced so as not to heat particles in the core orbit, essentially kicking them out of the machine. This condition was most prominent with a large number of particles in the core. The reduced gain of the stack tail system resulted in a decreased stacking rate. The betatron heating of the core is caused by a transverse component of kick given by the kicker electrodes. The transverse kick results from the fact that transverse pairs of kicker electrodes are not electrically identical and are not centered with respect to the beam.

To reduce the transverse kick, the entire kicker tank is aligned with respect to the core orbit. The previous stack tail system consisted of twenty kicker arrays contained in three vacuum tanks. Each array was powered by a pair of TWTs. An exact cancellation of the transverse kick could not occur because the frequency response of the each TWT and the electrical center of each array was not identical. The upgraded system consists of sixteen arrays contained in eight vacuum tanks. Since the number of TWTs per vacuum tank is much lower, the variation of the transverse kick due to the variation in the frequency response of the TWT's was reduced.

The kicker arrays of the previous system were fabricated with three dimensional stripline electrodes [4]. The reproducibility of the electrodes was on the order of 0.030 inch. The electrodes were suspended mechanically into a tube of rolled stainless steel. Due to the mechanical nature of the rolled stainless steel tubes, the alignment of the arrays to beam center varied by as much as \pm 0.020 inch along the length of the array.

As part of the upgrade, the three dimensional arrays were replaced with planar loop arrays. Planar loops were developed for use in Bunched Beam Stochastic Cooling in the TEVATRON [5]. The loops are fabricated on one side of a printed circuit board and the combiner board is placed on the other side. Because the arrays are etched using printed circuit techniques, the reproducibility of the electrodes in on the order of 0.001 inch. Since the printed circuit material is semiflexible it can be supported with a machined aluminum backing to obtain an accuracy of alignment on the order of 0.001 inch. In addition, since the electrodes and the electrode combiner are on the same circuit card, the arrays can be made substantially smaller.

As in the previous system, four sets of kicker electrodes were wired in the transverse mode to compensate for the transverse kick of the rest of the kicker tanks.. Each set corresponds to a different transverse plane and a different position in the lattice. The four sets of transverse electrodes are gain and phase adjusted to minimize the net transverse kick given by the entire stack tail system. However, since the frequency response of electrodes wired in the transverse mode is different from the net transverse kick of all the other electrodes, the transverse kick is not eliminated but is reduced by approximately $10 \mathrm{~dB}$. Because the number of tanks was increased from three to ten, the space allocated for vacuum flanges and bellows in between tanks was increased. To accommodate for this added space, four kicker arrays were sacrificed in the upgrade. However, in the upgrade, the transverse compensating kickers were also driven in the sum mode to provide extra longitudinal kick. Thus the number of longitudinal kicker arrays before and after the upgrade was kept constant. The sum and difference mode hybrid used to conbine the transverse and longitudinal kick had to have superb balance 
in order to not add extra unwanted transverse kick from the sum mode ports[3].

\section{NOTCH FILTERS}

The original notch filters included the Traveling Wave Tubes (TWT's) as part of the third notch filter. This presented operational difficulty as the gain and phase of the TWT's and their driver amplifiers had to be very carefully matched. Every time one of these components required replacement, there was degradation in filter \#3 performance, i.e. there is no such thing as an exact replacement. The original reason for doing this was to reduce odd order intermodulation products. Careful study showed that the reduction in intermodulation distortion did not warrant the added complexity of the filter. Because of the added active components within the correlator, vector gain balance was compromised and maximum notch depth was only 15 to $20 \mathrm{~dB}$.

The new notch filters are three passive BAW devices that require only temperature stabilization for reliable performance. The number of kicker TWT's has been reduced from 40 to 16 further increasing system reliability. The maximum notch depth is 25 to $40 \mathrm{~dB}$ for this filter which is a marked improvement over the previous filter.

\section{SYSTEM EQUALIZER}

With the availability of microwave simulation software [6] we were able to design a custom system equalizer to improve system bandwidth. An open loop measurement of the system was made that included both the electronic and beam transfer functions. This data was entered into the optimization software to develop a custom equalizer. The circuit is a stripline design that incorporates quadrature hybrids, coupled lines, and a Schiffman phase equalizer. Figure 1 shows the improvement in cooling bandwidth with the equalizer.

\section{SYSTEM PERFORMANCE}

Shortly after commissioning the new system, there was some skepticism as to how well it was functioning. Initial stacking rates were only $80 \%$ to $90 \%$ of the old system. After a couple of months of tuning the entire accumulator complex, stacking rates have exceeded $4.5 \times 10^{10}$ per hour. Stacking rate is about double the best rate of the original system and closing in on the design book value of $1.0 \times 10^{11}$ per hour. The current maximum stack size is $1.45 \times 10^{12}$. The design book value for this parameter was $4 \times 10^{11}$. The production efficiency of pbars from protons is consistently in the neighborhood of 15 parts per million now as opposed to 10 to 12 parts per million before the upgrades. (Figure 2) This number folds in all factors of accumulation showing a definite improvement in system nerformance. For comparison, 198889 collider run performance was a stack rate of $1.6 \times 10^{10}$ per hour and a production efficiency of 6 parts per million. Figure 3 shows a comparison of stacking statistics for the present run and that of the $1988-89$ collider run.
The main injector era requires improvement of source stacking rate to $1.5 \times 10^{11}$ per hour and stack sizes of $2 \times$ 1012. At this time it is not clear if the present system can handle these requirements. Eighty-five percent of all pbars produced at the target are presently stacked. The main injector may eventually more than double the amount of available pbars hence potentially saturating the system. Before the end of the current run, we are proposing reversal of magnet polarity so that a careful study of stacking protons can be made in an attempt to find the maximum stack rate of the present system. (Targeting protons can easily simulate the expected new Pbar production rate.)

The improvement in stacking of pbars is also due to improvements made in the debuncher cooling systems. Since 1987, a new debuncher momentum cooling system has been installed and the transverse cooling systems have had their power doubled. Also the Bunch Rotation voltage of the Debuncher has been increased to $5.1 \mathrm{MVolts}$. The main ring is once again supplying $2 \times 10^{12}$ protons per pulse to the target station.

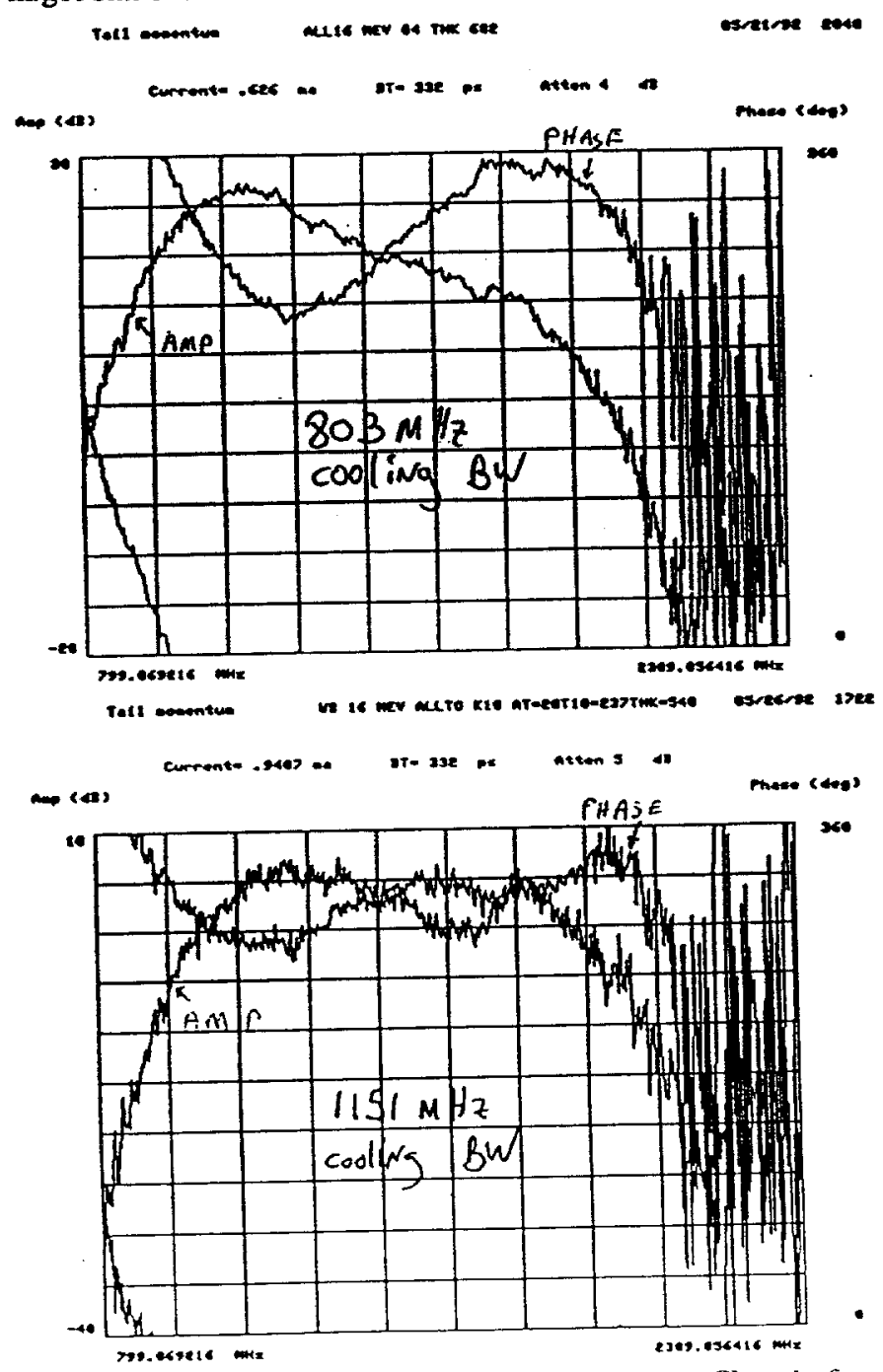

Figure 1. Open loop beam transfer function. Top: before equalizer system calculated cooling bandwidth is $803 \mathrm{MHz}$. Botom: after equalizer system calculated cooling bandwidth is $1151 \mathrm{MHz}$. 


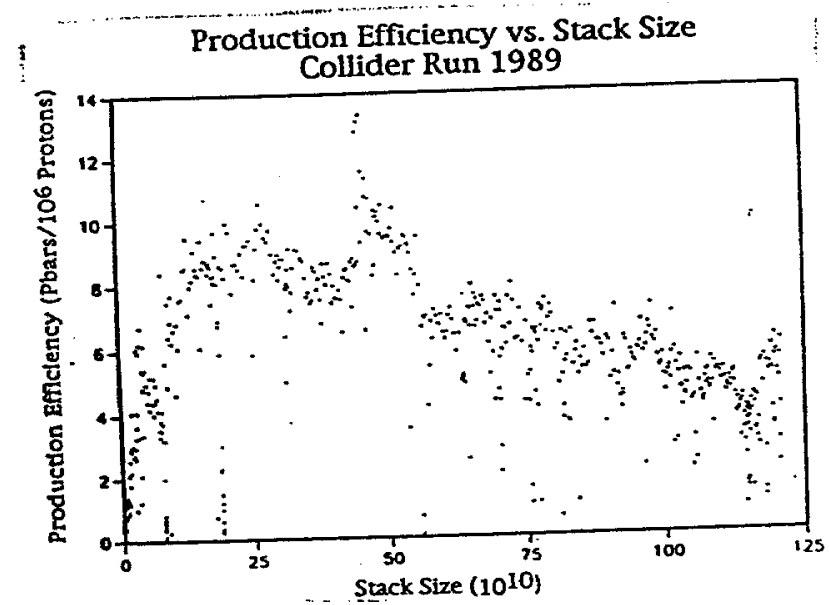

Production Efficiency vs. Stack Size

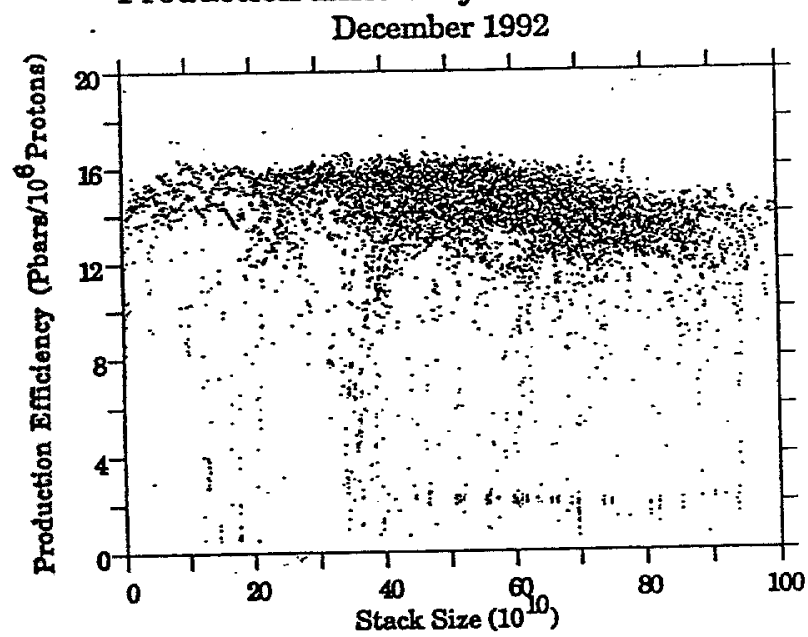

Figure 2. Comparison of production efficiency between 1989 collider run and present 1992-93 run.

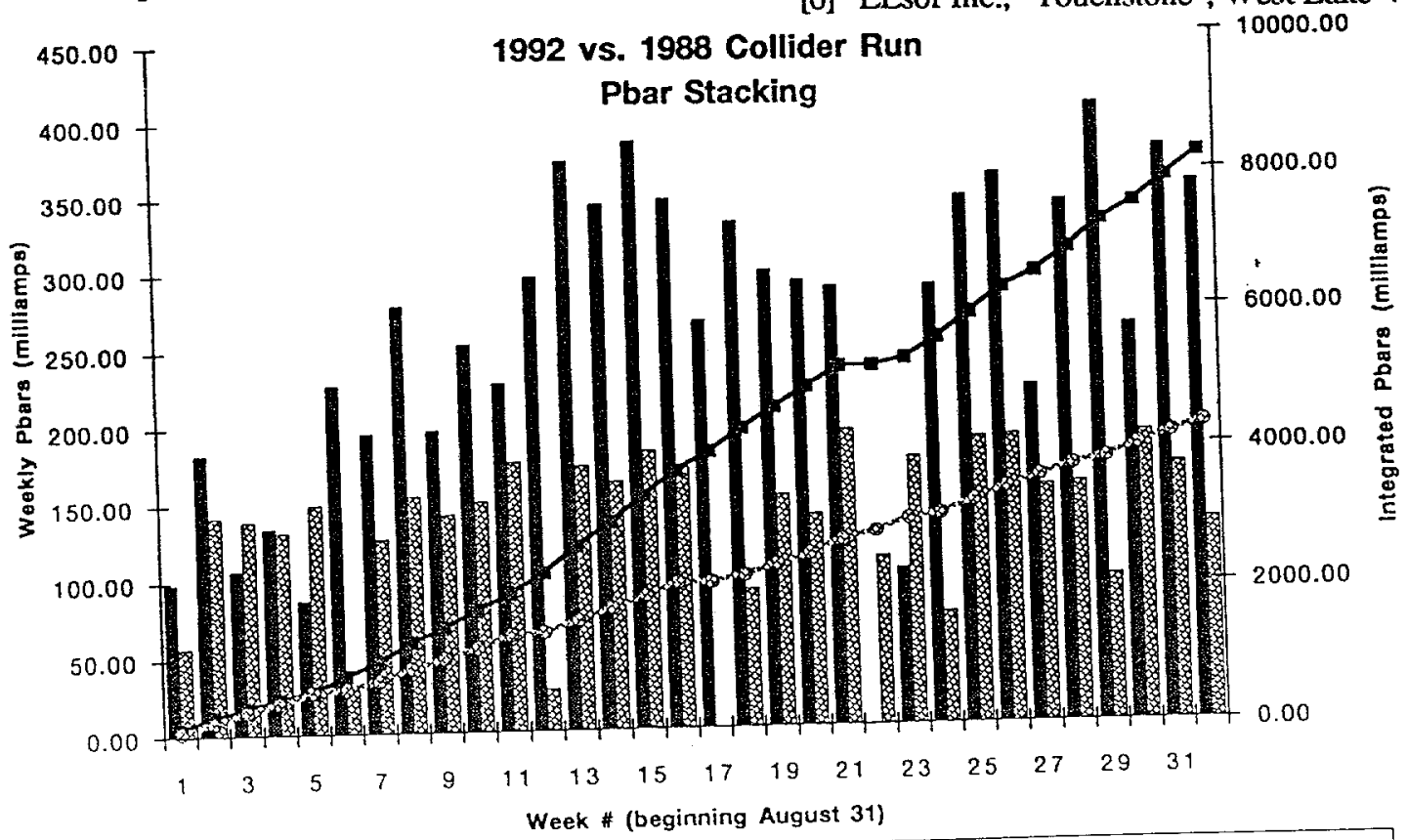

6ixis 1992 wkly Pbars 1988 Wkly Pbars
The goal for peak luminosity in the Tevatron for the present collider run was $5 \times 10^{30}$. Due in large part to the ability to accumulate more antiprotons in a shorter time that goal was shattered. The present peak luminosity achieved is 9 $\times 10^{30}$ with new records being achieved weekly.

\section{ACKNOWLEDGMENTS}

The improvements to the cooling system were a team effort by the members of the Antiproton source group, the mechanical support group, and other members of the Accelerator Division.

\section{REFERENCES}

[1] R.J. Pasquinelli , et al., "Stacktail Momentum Cooling in the Fermilab Antiproton Source", IEEE PAC 1987, Volume 2 pp. 1132-1134.

[2] R.J. Pasquinelli, "Bulk Acoustic Wave (BAW) Devices for Stochastic Cooling Notch Filters, IEEE PAC 1991, Volume 3, pp. 1395-1397.

[3] J. Petter, "Planar Slot Coupled Microwave Hybrids" U.S. Patent \# 5,075,647. December 1991.

[4] F. Voelker et al. "An Array of 1-2 GHz Electrodes for Stochastic cooling", IEEE Trans Nuclear Science NS-30, 1983, pp. 2262-2263

[5] D. McGinnis et. al., "Design of 4-8 GHz Bunched Beam Stochastic Cooling Arrays for the Fermilab Tevatron", IEEE PAC 1991, Volume 3, pp.13891391.

[6] EEsof Inc., "Touchstone", West Lake Village ,CA. 